\title{
Editorial
}

\section{Postoperative pain management - back to basics}

At first glance a paper describing the efficacy of intramuscular (im) analgesia is hardly headline material. But a closer look at the cost of im morphine makes this article very headline worthy, because it's cheap! Given the recent popularity of other more complex, more invasive and more expensive analgesic techniques, we may have forgotten just how simple, safe and effective im morphine can be. Given the relentless bombardment by newspaper headlines telling us that tough measures are needed to curb our escalating debt, we cannot ignore the importance of cost. Butscher's paper highlights the need for technology assessment in postoperative pain management, to examine the issues of both efficacy and cost.

\section{Efficacy}

Nurse-administered im opioid analgesia has long been the cornerstone of postoperative analgesia. While other techniques such as patient-controlled analgesia (PCA) and epidural analgesia have become popular, the nurse and the needle remain ubiquitous. Unfortunately, the practice of nurse-administered opioids has become synonymous with inadequate analgesia and is notoriously summarized as; "too little, too late." The solution is straightforward; give more, and give it more often. The most important reason for inadequate analgesia is the excessively long dosing interval created by the three letters "prn." The nurses are waiting for the patients to call, while the patient is waiting for the nurse to come. Just deleting these three letters "prn" from the analgesic prescription has a major impact on postoperative pain, since patients are guaranteed analgesic medication at least every four hours. Providing rescue analgesia for breakthrough pain further improves patient comfort. Multimodal analgesia is even better. ${ }^{2}$

Rather than adopt simple solutions we have popularized a sophisticated and expensive solution - PCA. The reason PCA is so effective is that it incorporates both

From the Department of Anaesthesia, University Hospital, University of Western Ontario, London, Ontario. of these solutions - more morphine, more often. The typical prescription for nurse-administered opioid analgesia is morphine $10 \mathrm{mg}$ im $\mathrm{q} 4 \mathrm{~h} \mathrm{prm}$. The typical prescription for PCA is morphine $1 \mathrm{mg} \mathrm{q6-10} \mathrm{min} \mathrm{prn} \mathrm{and} \mathrm{PCA}$ can provide as much as $40 \mathrm{mg}$ in four hours. Patients receiving PCA have access to more morphine. It is intriguing that we are more generous with our analgesic prescriptions when the patient is in control. It suggests that we have more confidence in the patient's ability to titrate opioid analgesia safely. Yet, the patient receives limited teaching, for a new technique, used in the early postoperative period, when cognitive function is considerably impaired. In contrast, the nurse has at least a two-year diploma, and may have years of experience, administering thousands of doses of opioids. Is the novice patient, with impaired cognitive function really more capable than the veteran nurse, with all faculties intact? If we allowed nurses these same dosing limits, wouldn't the results be very similar? After all, morphine is morphine.

Patient-controlled analgesia offers something more than just morphine. ${ }^{3}$ It offers the patients the security of knowing that they have some control over their therapy. Also, PCA guarantees that the patient is checked every hour, and if pain is not under control the nurse has immediate access to the Acute Pain Service where help "is only a phone call away." If all patients were assessed every hour, and if nurses were not hesitant to call surgeons for problems of inadequate analgesia, how different would these two therapies be? The important element of PCA is that the patient is in "control." All patients must be allowed to "control" their analgesic therapy by regular patient-centred pain assessments. A pain "flow sheet" could be a simple and effective addition to every bedside chart. Patient satisfaction with pain management may be more dependent upon the compassion with which it was delivered, than the efficacy of the medication. ${ }^{4}$

Butscher estimated that only $10 \%$ of patients after major surgery require epidural or patient-controlled analgesia. Other centres agree - these techniques are not necessary and are too expensive for routine use, and they 
are reserved for only $10-25 \%$ of surgical patients. ${ }^{5} \mathrm{Pi}$ oneers in PCA intended this therapy for patients with severe pain, that could not be managed by the nurse and the needle. ${ }^{6}$ Initially, it was estimated that three pumps would be needed for every 100 beds. ${ }^{7}$ Now, PCA has become a routine, first line therapy and is prescribed for up to $80 \%$ of surgical inpatients in both teaching ${ }^{8}$ and community hospitals. ${ }^{8,9}$ Some hospitals have a pump for almost every surgical patient. Some hospitals have more pumps than patients who need them. Patientcontrolled analgesia is even offered to patients after appendectomy, ${ }^{8}$ and has also been used after outpatient surgery. This is a classic example of "technology creep." 10

\section{Cost}

In Canada we enjoy a level of health care too rich for our wallets. "The recent federal budget heralds the end of an era, and the beginning of a new age. Restructure, restrain, reduce will be the battle cry of the 90 's. Most Canadians favour these social programme reforms. Reforms mean cuts. The recent federal budget has been described as the most painful budget in Canadian history. The stage is set for tough ethical, even life and death decisions. In the race to reduce cost we must strive to protect essential services for patients who need them the most.

Health care spending must go down. Medicare was developed to protect sick people from bankruptcy, not to provide them with every conceivable health care commodity. Even Prime Minister Chretien has hinted at "back-to-basics," "no-frills" health care and in the future universal medicare could be "leaner and meaner". ${ }^{12}$ For every $\$ 100$ produced by the Canadian economy $\$ 10$ is spent on health care and the Prime Minister says "that's too much. ${ }^{13}$ Conflict between cost and quality has only just begun. Provincial budgets will respond to the current federal budget and future federal budgets are expected to continue spending cuts. It may be too late for cost effectiveness. Cost containment and cost minimization are here to stay.

The cost-effectiveness of routine PCA is controversial. There is little evidence to support any reduced morbidity, reduced length of stay, or reduced hospital costs. ${ }^{8}$ Staffing patterns for surgical ward nurses may be more dependent on the pattern of admissions, and individual patient characteristics, than anaesthesia techniques ${ }^{14}$ or methods of postoperative pain management. Patient discharge patterns may be more dependent on geographic and social realities than physiological recovery. The patient may be ready for discharge, but the family may not. Unless we can simultaneously discharge the nurse with the patient, savings may be impossible to achieve. The savings must be real, not just theoretical.
To provide $24 \mathrm{hr}$ of pain relief for patients with nurseadministered im analgesia costs approximately $\$ 3$ per day. Oral morphine can also provide excellent analgesia and is even cheaper, only 48 cents per day. ${ }^{15}$ (Pain scores for oral morphine ${ }^{15}$ and PCA morphine ${ }^{16}$ are similar following hip arthroplasty.) Optimal multimodal, nurseadministered analgesia can be provided for only $\$ 3$ to $\$ 4$ per patient. ${ }^{5}$ Analgesia provided by an acute pain service may cost as much as $\$ 200$ to $\$ 300$ per patient. ${ }^{5}$ PCA may cost $\$ 33$ for supplies alone, ${ }^{9}$ plus the cost of salary support for members of the Acute Pain Service and physicians fees. ${ }^{17}$ Physician billing is an integral part of any pain service and may be the most expensive component.

Resources spent in any one area are resources taken from another. Health care dollars must be allocated to provide the greatest impact on the quality of life for a greater number of Canadians. We make similar economic choices in our personal affairs. We may not choose to purchase the most expensive car, even if it is safer and better, simply because it is far too expensive. In health care there are items which are more effective and more expensive. We must harness technology assessment to determine which modalities are basic essentials and which are unnecessary frills. Patients may like these frills, and they may want them, but do they need them? If patients had to pay, would they? Could they?

\section{Summary}

Butscher describes a common-sense approach to pain management which is simple, safe and effective. A wide variety of national and international organizations devoted to the management of pain have universally adopted these simple measures. Current guidelines advocate both regular dosing and rescue analgesia. These guidelines were produced by an army of international experts and have been available for a decade. Although the information is widely published, it is rarely adopted in clinical practice. Is it any wonder that leaders in the field of pain management ask: "Is education enough?"19 "Will guidelines make a difference?"20 They continue to lament the "tragedy of needless pain"21 and in despair they have called for "national initiatives on pain."

Bonica stated so elegantly, "for nearly 30 yr I have studied the reasons for inadequate management of postoperative pain, and they remain the same ... inadequate or improper application of available information and therapies is certainly the most important reason for inadequate postoperative pain relief. ${ }^{23}$ Butscher's data support Bonica's theory that the problem of inadequate analgesia does not require futuristic high tech solutions. In fact, as the economic crunch continues we may find that we cannot afford some of these new, improved and 
more expensive techniques. If we can provide safe and effective analgesia for only pennies a day, this option cannot be ignored. ${ }^{15}$ The new way may be the old way.

\section{L'analgésie postopératoire: revenons à l'essentiel}

Au premier abord, un article qui sintéresse à l'efficacité analgésique de la morphine intramusculaire peut difficilement faire l'objet d'une manchette. Mais si on y regarde de plus près, la morphine im constitue l'occasion d'une manchette sensationnelle, parce qu'elle ne coûte presque rien. En face de la popularité de techniques plus complexes, plus effractives et plus chères, on semble avoir oublié la simplicité, l'efficacité et la sécurité que procure la morphine im. Alors que dans les journaux, nous nous faisons répéter inlassablement que des mesures drastiques doivent être prises pour enrayer la croissance de notre endettement, nous ne pouvons ignorer les coûts inhérents à nos actes. L'article de Butscher souligne la nécessité de l'évaluation méthodique du traitement de la douleur postopératoire tant sous l'aspect de son coût que de son efficacité.

\section{L'efficacité}

L'analgésie morphinique im fut pendant longtemps la pierre angulaire de l'analgésie postopératoire. Alors que d'autres techniques telles que l'analgésie contrôlée par le patient (PCA) et l'analgésie épidurale sont devenues à la mode, l'infirmier/ère et son aiguille sont toujours omniprésents. Malheureusement, l'administration d'un morphinique par le personnel infirmier est devenue synonyme d'insuffisance analgésique et a été étiquetée sous la formule " trop peu, trop tard ". ${ }^{1}$ La solution est pourtant très simple: en donner plus, et plus souvent. La raison la plus importante de la médiocrité de l'analgésie est constituée par l'excessive longueur de l'intervalle représenté par les lettres " p.r.n. ". Le personnel infirmier attend que le patient appelle alors que le patient attend que l'infirmier/ère vienne à lui. Le seul fait d'omettre ces trois lettres " p.r.n. " dans l'ordonnance a un impact important sur la douleur, parce qu'on assure au patient qu'il recevra un analgésique à toutes les quatre heures au moins. En outre, si on lui procure une analgésie de sauvetage contre les percées douloureuses, on améliore son bien-être. Une analgésie qui combine plusieurs technique est encore meilleure. ${ }^{2}$

Plutôt que d'avilir recours à la solution la plus simple, nous avons adopté la plus sophistiquée et la plus coûteuse - la PCA. La raison de l'efficacité de la PCA consiste en ce qu'elle incorpore les deux solutions mentionnées: plus de morphine, et plus souvent. L'ordonnance typique administrée par le personnel infirmier se lit ainsi morphine $10 \mathrm{mg}$ im q4h prn; en PCA, la morphine est prescrite $1 \mathrm{mg} \mathrm{q6-10} \mathrm{min} \mathrm{prn.} \mathrm{Par} \mathrm{la} \mathrm{PCA,} \mathrm{le} \mathrm{patient} \mathrm{peut}$ recevoir $\mathbf{4 0} \mathrm{mg}$ en quatre heures. Ces patients ont ainsi accès à une dose plus forte de morphine. Il est étrange de constater que nous sommes plus généreux dans nos prescriptions de morphiniques quand c'est le patient qui les contrôle. Nous pouvons donc conclure que nous faisons plus confiance à nos patient au regard de sa sécurité parce que nous lui confions la responsabilité de doser lui-même ses morphiniques. Toutefois, avec cette nouvelle technique, le patient reçoit peu d'instruction, et lorsqu'il l'utilise à la période postopératoire immédiate, sa fonction cognitive est de beaucoup réduite. Au contraire, l'infirmière a étudié pendant au moins deux ans, et possède souvent plusieurs années d'expérience et a déjà administré des morphinique des milliers de fois. Est-ce que le néophyte avec des facultés affaiblies est réellement plus apte que linfirmière chevronnée en pleine possession de toutes ses facultés? Il ne faut pas l'oublier, la morphine demeure de la morphine.

La PCA offre plus que de la morphine. ${ }^{3}$ Elle permet au patient conscient d'exercer avec sécurité un certain contrôle sur son soulagement. Egalement, la PCA assure que le patient sera revu à toutes les heures et que si la douleur n'est pas maîtrisée, l'infirmier/ère a un accès immédiat à un service de traitement de la douleur aiguë qui vient à son secours presque instantanément. Si tous les patients revus à chaque heure, et si le personnel infirmier nhésitait pas à rappeler le chirurgien pour qu'il prescrive une analgésie adéquate, quelle serait la différence entre les deux approches? L'élément primordial de la PCA, c'est que le patient exerce un contrôle. Il faut permettre à tous les patients de contrôler leur analgésie et l'évaluer régulièrement. Un ordinogramme pour la douleur serait une addition simple et efficace au dossier de chevet. La satisfaction d'un patient en rapport avec son analgésie peut relever plus de la compassion avec laquelle elle est administrée que de l'efficacité de la médication elle-même. ${ }^{4}$

Butscher croit qu'après une chirurgie majeure, seulement $10 \%$ des patients ont besoin d'analgésie épidurale ou autocontrôlée. Plusieurs autres centres suivent une politique identique (ces techniques ne sont pas nécessaires et coûtent trop cher pour une utilisation en routine) et ne les réservent qu’à $10-15 \%$ des patients chirurgicaux. ${ }^{5}$ 
Les pionniers de la PCA destinaient cette thérapie aux grandes douleurs, celles qui ne pouvaient être contrôlées par linfirmier/ère et son aiguille. ${ }^{6}$ Originalement, on calculait que trois pousse-seringue seraient nécessaires pour 100 lits. $^{7}$ Maintenant, la PCA est devenue une habitude, un traitement de première ligne, et est prescrite pour près de $80 \%$ des patients hospitalisé aussi bien dans les hôpitaux d'enseignement ${ }^{8}$ que dans les autres. ${ }^{8,9}$ Certains hôpitaux possèdent un pousse-seringue pour chacun des lits chirurgicaux. D'autres ont plus de pompes que de patients chirurgicaux. La PCA est même offerte aux appendicectomisés, ${ }^{8}$ et parfois même aux patients ambulatoires. Ceci représente un exemple classique de l'aberration technologique. ${ }^{10}$

\section{Coût}

Au Canada, nous jouissons d'un système de santé devenu trop cher pour nos moyens. ${ }^{11}$ Le récent budget fédéral annonce la fin d'une époque et le début d'un âge nouveau. Restructurations, retenues et coupures seront les cris d'appel au combat des années 90 . La plupart de Canadiens sont en faveur de la réforme des programmes sociaux. Réforme signifie coupure. On a décrit le dernier budget fédéral comme le plus douloureux de l'histoire canadienne. La scène est mise pour les choix d'éthique difficiles même sur les question de vie et de mort. Dans notre marche vers la réduction des coûts, nous devons nous efforcer de défendre les services essentiels pour les patients qui en ont le plus besoin.

Les coût des soins de santé doivent baisser. L'assurance-santé a été créée pour protéger les malades de la banqueroute, non pour les pourvoir de toutes les facilités imaginables en soins de santé. Même le premier ministre Chrétien a laissé présager un retour aux soins santé de base, san parures, et a énoncé que dans la future l'universalité des soins sera plus mince et plus chiche. ${ }^{12}$ Pour chaque cent dollars dépensés, dix le sont pour la santé et le premier ministre juge que c'est trop. ${ }^{13} \mathrm{Le}$ conflit entre le coût et la qualité ne fait donc que commencer. Les budgets provinciaux suivront la voie tracée par le budget fédéral et il faut s'attendre à ce que les prochains budgets fédéraux continuent dans la mème veine. Il est peut-être déjà trop tard pour parler du coût en rapport avec l'efficacité. La maîtrise des dépenses et leur réduction sont ici pour y rester.

L usage de routine de la PCA est très discutable au regard du coût/efficacité. II n'est pas prouvé qu'elle réduit la morbidité, la durée du séjour et des coûts hospitaliers. L'attribution de postes aux infirmières de service en chirurgie dépend plus du type d'admission, et des caractéristiques du patient que des techniques anesthésiques ${ }^{14}$ ou des méthodes de traitement antalgique. Les critères qui gèrent le congé hospitalier dépendent plus des réa- lités géographiques et sociales que de la récupération physiologique. Le patient peut être prêt à quitter l'hôpital sans que sa famille soit prête à le recevoir. Il peut être impossible de réaliser des économies si on ne réussit pas à donner congé à linfirmier/ère en même temps que son patient. Les économies doivent être réelles, non seulement théoriques.

Pour procurer 24 heures de soulagement, l'administration de l'analgésie im par du personnel infirmier coûte trois dollars par jour. La morphine per os fournit une excellente analgésie et coûte encore moins cher, seulement 48 cents par jour. (Les scores de douleur pour la morphine orale 15 et la morphine en PCA sont les mêmes après une arthroplastie de la hanche). Une analgésie optimale, associant plusieurs techniques, administrée par le personnel infirmier peut être réalisée pour seulement trois à quatre dollars par jour par patient. ${ }^{5} \mathrm{~L}$ 'analgésie procurée par un service de traitement de la douleur aiguë coûte autant que $200 \$$ à $300 \$$ par patient. ${ }^{5}$ La PCA coûte $33 \$$ pour le matériel seulement, plus le salaire des membres du service de la douleur aiguë et les honoraires professionnels. ${ }^{17}$ Les honoraires des médecins font partie intégrale de tout service anti-douleur et représentent souvent la plus grande part des coûts.

Les ressources dépensées dans un domaine sont des ressources dont un autre est privé. Les dollars attribués aux soins de santé doivent être répartis de façon à produire le plus grand impact possible pour le plus grands nombre de Canadiens possible. C'est bien là le type de choix que nous faisons pour nos affaires personnelles. Nous ne choisissons pas nécessairement la voiture le plus chère, parce qu'elle coûte trop cher, mème si elle offre une plus grande sécurité et une meilleure qualité. Nous devons évaluer la technologie pour en déterminer le part de l'essentiel et ce qui constitue des accessoires superflus. Le patient peut aimer ces garnitures et mème les désirer mais en a-t-il réellement besoin? Si la patient avait à payer, le ferait-il? Le pourrait-il?

\section{Résumé}

Butscher décrit une façon sensée de gérer la douleur qui brille par sa simplicité, sa sécurité et son efficacité. Plusieurs organismes nationaux et internationaux dédiés à la gestion de la douleur déjà ont adoptés ces dispositions élémentaires. La ligne de conduite proposée préconise à la fois un titrage régulier du morphinique avec une analgésie de sauvetage. Ces directives ont été élaborées par une armée d'experts internationaux et sont promulguées depuis une décennie. Bien que cette information soit largement répandue, elle est rarement adoptée en clinique. N'est-il pas étonnant que les leaders dans le domaine de la douleur doivent encore sinterroger sur la déficience de l'enseignement ${ }^{19}$ et sur l'utilisation de directives. ${ }^{20} \mathrm{Il}$ 
est tragique de toujours répéter que la douleur est toujours inutile ${ }^{21}$ et en désespoir de cause de demander à nouveau un mobilisation nationale contre la douleur. ${ }^{22}$

Bonica a écrit que, pendant près de 30 ans, il avait étudié les causes de l'échec thérapeutique antalgique et qu'elles découlaient d'une fausse interprétation de nos connaissances et de l'application incorrecte de moyens à notre disposition; ce qui de toute de toute évidence explique l'échec du traitement de la douleur postopératoire. ${ }^{23}$ Les données de Butscher confirme la thèse de Bonica selon laquelle il n'est pas nécessaire de recourir à une technologie avancée et d'avant-garde pour solutionner le problème de la douleur mal soulagée. En réalité, soumis à une contrainte économique permanente, nous pourrions trouver inabordables quelques unes des techniques plus modernes, perfectionnées et aussi plus coûteuses. Si nous pouvons procurer une analgésie efficace en toute sécurité pour seulement quelques sous par jour pourquoi ne pas le faire? ${ }^{15}$ La nouvelle voie à suivre pourrait fort bien être l'ancienne.

\section{References}

1 Moote $C A$. The prevention of postoperative pain. Can J Anaesth 1994; 41: 527-33.

2 Moote $C$. Efficacy of nonsteroidal anti-inflammatory drugs in the management of postoperative pain. Drugs 1992; 44(Supplement 5): 14-29.

3 Ready LB. Patient-controlled analgesia - does it provide more than comfort? (Editorial). Can J Anaesth 1990; 37: 719-21.

4 Ward SE, Gordon D. Application of the American Pain Society quality assurance standards. Pain 1994; 56: 299-306.

5 Rawal N, Breggren L. Organization of an acute pain service: a low-cost model. Pain 1994; 57: 117-23.

6 Keeri-Szanto M, Heaman S. Postoperative demand analgesia. Surg Gynecol Obstet 1972; 134: 647-51.

7 Keeri-Szanto M. Demand analgesia (pain is a four-letter word). Pharmacy Bulletin, Drug Information Centre, Victoria Hospital Jan/Feb: 1, 1981.

8 Etches RC. Respiratory depression associated with patientcontrolled analgesia: a review of eight cases. Can J Anaesth 1994; 41: 125-32.

9 Friedlander $M$. The acute pain service: how to establish one in a community hospital. Contemporary Anaesthesia 1993; October: 13-9.

10 Naylor CD, Williams JI, Basinski A, Goel V. Technology assessment and cost-effectiveness analysis: misguided guidelines? Can Med Assoc J 1993; 148: 921-4.

11 OReilly $M$. Too rich for our own blood? The London Free Press, 1995; E1.

$12 \operatorname{Cox} B$. And universal medicare could be leaner, meaner. The London Free Press, 1995: A3.
13 Free Press News Services: Chretien says Canada extravagant on medicare. The London Free Press, 1995; A1.

14 Dexter $F$, Tinker $J H$. Analysis of strategies to decrease postanaesthetic care unit costs. Anesthesiology 1995; 82 94-101.

15 McCormack JP, Warriner CB, Levine M, Glick N. A comparison of regularly dosed oral morphine and ondemand intramuscular morphine in the treatment of postsurgical pain. Can J Anaesth 1993; 40: 819-24.

16 Segstro $R$, Morley-Forster $P K, L u G$. Indomethacin as a postoperative analgesic for total hip arthroplasty. Can J Anaesth 1991; 38: 578-81.

17 Moote CA. Pharmacoeconomics of patient-controlled analgesia. Perspectives of Pain Management 1994; 3: 7-12.

$18 O M A$. Utilization-reduction mechanisms to be considered by Council at Feb meeting. The Medical Times 1995; 4: 1-2.

19 Max $M B$. Improving outcomes of analgesic treatment: is education enough? Ann Intern Med 1990; 113: 885-9.

20 Max MB. U.S. Government disseminates acute pain treatment guidelines: will they make a difference? (Editorial). Pain 1992; 50: 2-3.

21 Melzack $R$. The tragedy of needless pain. Scientific American 1990; 262: 27-33.

22 Janeti J, Liebeskind $J C$. A call for national initiatives on pain. Pain 1994; 59: 5-6.

23 Bonica JJ. The Management of Pain. Philadelphia: Lea \& Febiger, 1990. 